\title{
Sixth nerve palsy
}

\section{Mini review}

Among the ocular motor nerve palsies sixth nerve palsy is the most common followed by fourth nerve and third nerve. Acute palsies can pose a diagnostic and therapeutic challenge to the ophthalmologist. Abducens nerve is susceptible to damage due to various intracranial pathologies. This is because of its long intracranial path, its angle at the petrous tip of the temporal bone and non-flexibility of the path between the brainstem and the meningeal entrance site. Various causes include infections (meningitis, varicella zoster), trauma (head injury), neoplasms (chondroma, metastasis), systemic disorders (atherosclerosis, leukaemia) and iatrogenic (post spinal anaesthesia). Sixth nerve palsies can occur in both adults and children. Those occurring in adults above the age of 40 years are most commonly caused by atherosclerosis and microangiopathies like hypertension and diabetes mellitus. Patients with acute sixth nerve palsy present with incapacitating diplopia. Ocular motility in these cases improves in $94 \%$ of the patients by six months spontaneously. In general, at the onset of an isolated sixth nerve palsy in a vasculopathic patient, neuroimaging is not required. However, a cranial MRI is mandatory if obvious improvement has not occurred after 3 months if associated signs are present. Other diagnostic studies that may be required include lumbar puncture, chest imaging, and hematologic studies to identify an underlying systemic process such as collagen vascular disease, sarcoidosis, or syphilis.

In younger individuals under 50 years a careful neuroimaging is required because only a few cases are due to ischemic cranial neuropathy. If neuroimaging is negative consideration should be given to neuromuscular junction disease by obtaining acetylcholine antibodies or performing Tensilon testing. Mechanical pathophysiologies such as thyroid eye disease with medial rectus involvement and meningeal-based disease should be considered. In adolescents and young adults, demyelination (multiple sclerosis) may be the cause, and in such cases MRI with fluid -attenuated inversion recovery (FLAIR) imaging should be performed. During the first six months after the onset of palsy, conservative management in the form of patching, prisms or botulinum toxin injections are used to prevent diplopia and abnormal head posture. Long standing esotropia will lead to contracture of the medial rectus giving a positive forced duction test (FDT) which can be prevented by injecting botulinum toxin into the muscle. Surgical plan for a patient with sixth nerve palsy varies depending on the strength of the lateral rectus muscle (palsy or paresis). If preoperative FGT shows some residual LR function then medial rectus recession and lateral rectus resection $(R \& R)$ procedures can be carried out. In cases of complete sixth nerve palsy where there is no LR function on FGT then a transposition procedure has to be performed along with medial rectus recession (Figures 1\&2). Different muscle transposition procedures include Hummelsheim, Jensen and Nishida with or without augmentation procedures like Wright, Foster and Brooks.

In complete sixth nerve palsy, transposition procedure provides an abducting force to the paralysed muscle, increasing the diplopia free field with improved centration. Hummelsheim described the first
Volume 8 Issue I - 2018

\author{
Smita Kapoor, Rajesh Prabu, Swarna \\ Udayakumar \\ University Sankara Eye Hospital, India
}

Correspondence: Smita Kapoor, University Sankara Eye Hospital, Sathy Road, Coimbatore, India, Tel 9566632262 , Email smitakapoor21@yahoo.in

Received: January 03, 2018 | Published: January 18, 2018

rectus muscle transposition procedure for treating paralytic strabismus in 1908. Several modifications to his procedure were described by O'Connor, Jensen, Knapp, Carlson and Jampolsky. However, the original principle of all the surgeries remains the same. The principle involves shifting forces of antagonist muscles to a rectus muscle that lies between them and acting in the opposite plane. This provides an abducting force to the paralysed muscle. In Hummelsheim's procedure of partial tendon transposition for the paralytic lateral rectus, temporal halves of the superior rectus and inferior rectus were transposed to the paralysed lateral rectus muscle (Figures $3 \& 4$ ). Jenson described a rectus muscle union procedure in which the lateral half of the split SR and IR is united with the corresponding superior and inferior halves of the LR. It was combined with MR recession. It had the advantage of not disinserting the muscle and thereby sparing the anterior ciliary circulation. Still this technique had the risk of strangulation of the vessel when the rectus muscles were united. ${ }^{1,2} \mathrm{O}^{\prime}$ Connor performed a full tendon transposition of the SR and IR to the LR combined with cinch procedure on the LR. The disadvantage being more chances of vertical imbalance. However, due to the increased risk of anterior segment ischemia involved in all these procedures, Nishida et al proposed an effective procedure in which tenotomy was not performed..$^{3-5}$ In the procedure explained by the author the muscle belly of superior and inferior recti are longitudinally split and the lateral halves are transposed close to the superior and inferior border of the lateral rectus respectively $12 \mathrm{~mm}$ posterior to the insertion. (Figure 5) This procedure achieves the same postoperative results as other procedures. ${ }^{6}$ The laterally transposed muscle bellies generate additional abductional forces at the new points of insertion (Figure 6).

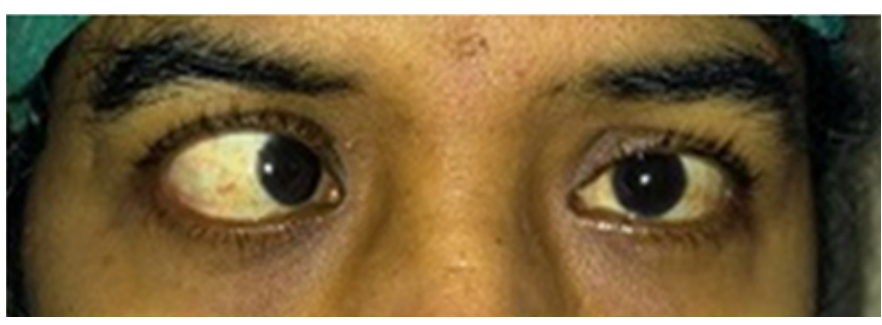

Figure I Preoperative photograph showing right eye esotropia. 


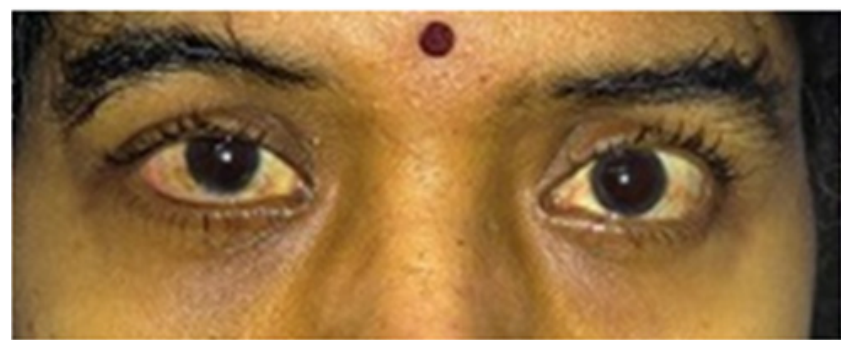

Figure 2 Postoperative photograph showing orthotropia following muscle transposition procedure.

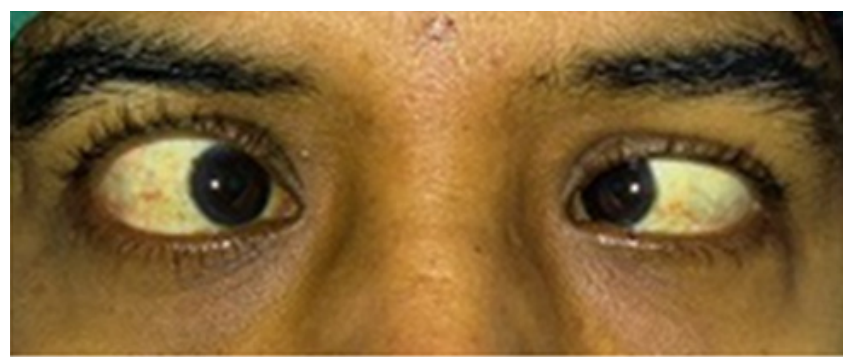

Figure 3 Preoperative photograph showing abduction limitation in right eye.

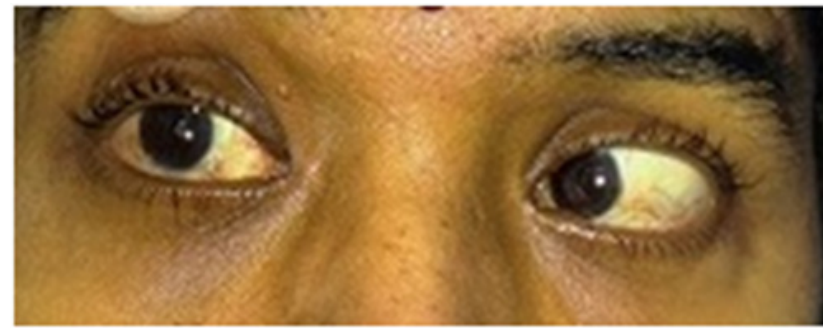

Figure 4 Postoperative photograph showing improvement in abduction in right eye.

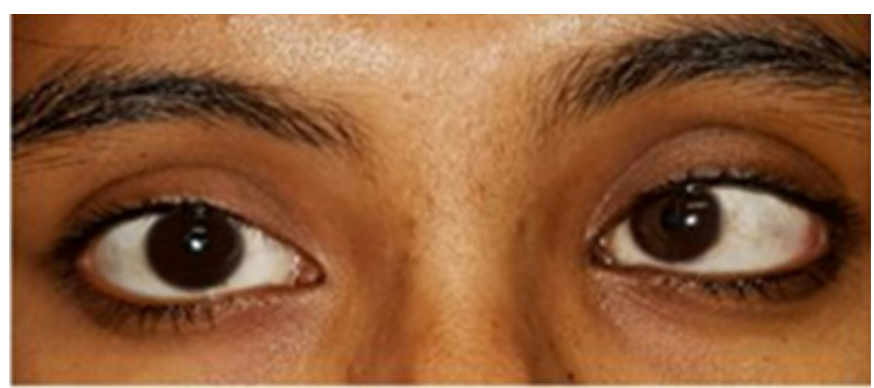

Figure 5 Preoperative photograph showing left eye esotropia.

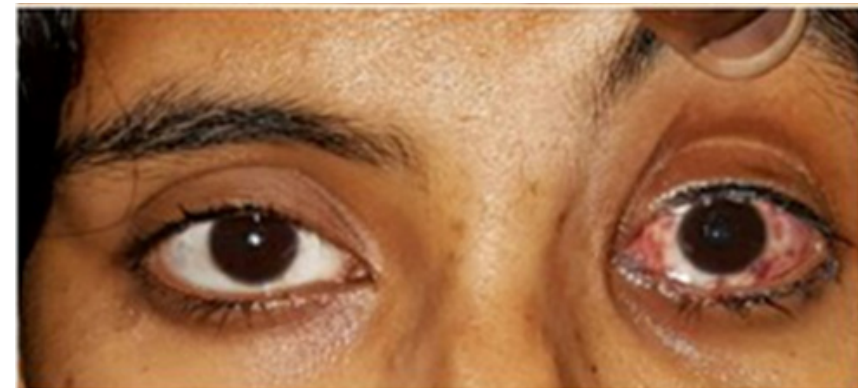

Figure 6 Postoperative photograph showing orthotropia following muscle transposition procedure.

\section{Acknowledgments}

None.

\section{Funding}

None.

\section{Conflicts of interest}

None.

\section{References}

1. Jensen CD. Rectus muscle union: a new operation for paralysis of the rectus muscles. Trans Pac Coast Otoophthalmol Soc Annu Meet. $1964 ; 45: 359-387$

2. Von Noorden GK. Anterior segment ischemia following the Jensen procedure. Arch Ophthalmol. 1976;94(5):845-847.

3. Tanaka M, Nishina S, Ogonuki S, et al. Nishida's procedure combined with medial rectus recession for large-angle esotropia in Duane syndrome. Jpn J Ophthalmol. 2011;55(3):264-267.

4. Nishida Y, Hayashi O, Oda S, et al. A simple muscle transposition procedure for abducens palsy without tenotomy or splitting muscles. Jpn J Ophthalmol. 2005;49(2):179-180.

5. Nishida Y, Inatomi A, Aoki Y, et al. A muscle transposition procedure for abducens palsy, in which the halves of the vertical rectus muscle bellies are sutured onto the sclera. Jpn J Ophthalmol. 2003;47(3):281-286.

6. Muraki S, Nishida Y, Ohji M . Surgical results of a muscle transposition procedure for abducens palsy without tenotomy and muscle splitting. Am J Ophthalmol. 2013;156(4):819-824. 